\title{
Human Ex vivo Wound Model and Whole-Mount Staining Approach to Accurately Evaluate Skin Repair
}

\author{
Holly Nicola Wilkinson ${ }^{1}$, Alexandria Sarah Kidd ${ }^{1}$, Elizabeth Rose Roberts ${ }^{1}$, Matthew James Hardman ${ }^{1}$ \\ ${ }^{1}$ Centre for Atherothrombosis and Metabolic Disease, Hull York Medical School, University of Hull
}

\section{Corresponding Author}

Holly Nicola Wilkinson

h.n.wilkinson@hull.ac.uk

\section{Citation}

Wilkinson, H.N., Kidd, A.S.,

Roberts, E.R., Hardman, M.J. Human Ex vivo Wound Model and WholeMount Staining Approach to Accurately Evaluate Skin Repair. J. Vis. Exp. (168), e62326, doi:10.3791/62326 (2021).

\section{Date Published}

February 17, 2021

DOI

$10.3791 / 62326$

URL

jove.com/video/62326

\section{Abstract}

Chronic non-healing wounds, which primarily affect the elderly and diabetic, are a significant area of clinical unmet need. Unfortunately, current chronic wound treatments are inadequate, while available pre-clinical models poorly predict the clinical efficacy of new therapies. Here we describe a high throughput, pre-clinical model to assess multiple aspects of the human skin repair response. Partial thickness wounds were created in human ex vivo skin and cultured across a healing time course. Skin wound biopsies were collected in fixative for the whole-mount staining procedure. Fixed samples were blocked and incubated in primary antibody, with detection achieved via fluorescently conjugated secondary antibody. Wounds were counterstained and imaged via confocal microscopy before calculating percentage wound closure (re-epithelialization) in each biopsy. Applying this protocol, we reveal that $2 \mathrm{~mm}$ excisional wounds created in healthy donor skin are fully re-epithelialized by day 4-5 post-wounding. On the contrary, closure rates of diabetic skin wounds are significantly reduced, accompanied by perturbed barrier reformation. Combining human skin wounding with a novel whole-mount staining approach allows a rapid and reproducible method to quantify ex vivo wound repair. Collectively, this protocol provides a valuable human platform to evaluate the effectiveness of potential wound therapies, transforming pre-clinical testing and validation.

\section{Introduction}

Chronic, non-healing wounds, which are highly prevalent in the elderly and diabetic, are a majorly unappreciated area of clinical unmet need. These wounds present a major physical and psychological burden to patients and cost healthcare providers billions each year to treat ${ }^{1}$. Despite improved understanding of wound biology and advancements in technology, up to $40 \%$ of chronic wounds still fail to heal following best standard care ${ }^{2}$. Thus, $14-26 \%$ of patients with diabetic foot ulcers subsequently require amputation ${ }^{3}$, while 5-year post-amputation mortality rate stands at approximately $70 \%{ }^{4}$. As a result, there is an urgent requirement to develop efficacious new therapies to improve patient quality of life 
while reducing the substantial healthcare burden imposed by poor healing wounds. Poorly predictive pre-clinical models remain a significant hurdle to the development of effective new therapies.

Wound repair is a dynamic and multifaceted process involving a diverse range of cell types, countless levels of communication and a tissue environment that is temporally remodeled. Skin healing is underpinned by four major reparative stages: hemostasis, inflammation, proliferation, and matrix remodeling. These stages ultimately act to prevent blood loss and infection, close the wound surface (a process termed re-epithelialization) and return the skin to an uninjured state ${ }^{5}$. Chronic wounds are associated with diverse etiology and widespread perturbation to healing processes ${ }^{6}$, further complicating the identification of therapeutic targets. Nevertheless, a broad range of models have been developed to both elucidate the molecular and cellular drivers of wound pathology and test new therapeutic approaches ${ }^{7}$.

The most used wound repair model is acute wounding in the mouse. Mice are highly tractable for mechanistic studies and provide validated models of ageing and diabetes ${ }^{8}$. Despite the general similarities shown amongst mouse and human healing, between-species differences in skin structure and healing dynamics remain. This means most murine wound research does not easily translate to the clinic $^{9}$. Consequently, there has been a push towards human in vitro and ex vivo systems with high applicability and translatability 10,11 .

Here we provide an in-depth protocol for performing partial thickness excisional wounds in ex vivo human skin. We also outline our whole-mount staining approach as a highly reproducible method of evaluating ex vivo human skin healing. We show the trajectory of epidermal repair (re-epithelialization) and subsequent barrier formation, evaluating the rate of wound closure in healthy versus diabetic human skin. Finally, we demonstrate how wholemount staining can be adapted for use with a range of antibodies to assess various aspects of the healing response.

\section{Protocol}

Human skin was obtained from patients undergoing reconstructive surgery at Castle Hill Hospital and Hull Royal Infirmary (Hull, UK) under full informed, written patient consent, institutional guidelines, and ethical approval (LRECs: 17/SC/0220 and 19/NE/0150). Non-diabetic skin was collected from patients undergoing routine surgery (mean age $=68)$. Diabetic skin was selected from donors who had established type II diabetes and a history of ulceration (mean age $=81)$. Samples from surgery were transported in holding media and processed immediately upon arrival at the laboratory. All experimental steps using unfixed human tissue were performed at Biosafety Level-2 (BSL-2) in a class Il laminar flow biosafety cabinet.

\section{Preparation of skin culture media and staining reagents}

NOTE: All reagent and consumable details are provided in the Table of Materials. Ensure all reagents and equipment used for the processing and culture of human tissue are sterile. Sterilize instruments prior to the use and decontaminate with disinfectant following contact with the tissue. Decontaminate waste products in $1 \%$ disinfectant before disposal.

1. Holding media: Supplement high glucose Dulbecco's Modified Eagle Medium (DMEM) with $2 \mathrm{mM}$ L-glutamine and 4\% (v/v) antibiotic-antimycotic solution. 
2. Hank's balanced salt solution (HBSS) with antibiotics: Add 4\% (v/v) antibiotic-antimycotic solution to HBSS. Store at $4{ }^{\circ} \mathrm{C}$ until use.

3. Dulbecco's phosphate buffered saline (DPBS): Prepare DPBS by dissolving $9.6 \mathrm{~g}$ of DPBS powder per liter of distilled water $\left(\mathrm{dH}_{2} \mathrm{O}\right)$. Autoclave to sterilize and store at $4{ }^{\circ} \mathrm{C}$ until use.

4. Human skin growth media: Supplement high glucose DMEM with $2 \mathrm{mM}$ L-glutamine, 1\% (v/v) antibioticantimycotic solution and $10 \%(\mathrm{v} / \mathrm{v})$ fetal bovine serum. Store at $4{ }^{\circ} \mathrm{C}$ until use.

5. Skin fixative: To $450 \mathrm{~mL}$ of $\mathrm{dH}_{2} \mathrm{O}$, add $40 \mathrm{~mL}$ of formaldehyde solution, $10 \mathrm{~mL}$ of glacial acetic acid, $4.5 \mathrm{~g}$ of sodium chloride and $0.25 \mathrm{~g}$ of alkyltrimethylammonium bromide. Store at room temperature (RT) and use within a few days.

CAUTION: Fixative is hazardous (irritant and flammable). Handle with care and dispose of via an appropriate route.

6. Phosphate buffered saline (PBS): Prepare PBS for whole-mount staining by adding $6 \mathrm{~g}$ of sodium chloride to $100 \mathrm{~mL}$ of phosphate buffer solution and $900 \mathrm{~mL}$ of $\mathrm{dH}_{2} \mathrm{O}$.

7. Staining wash buffer: Dissolve $0.5 \%(\mathrm{v} / \mathrm{v})$ Triton $\mathrm{X}-100$ in PBS.

8. Blocking buffer: Add $0.2 \%(\mathrm{w} / \mathrm{v})$ sodium azide and $2 \%(\mathrm{v} /$ v) animal serum to staining wash buffer. Store at $4{ }^{\circ} \mathrm{C}$ for up to two weeks.

NOTE: Block in the serum of the secondary antibody host species. Sodium azide will prevent bacterial growth during incubation.

9. DAPI working solution: Prepare a $5 \mathrm{mg} / \mathrm{mL}$ stock of $4{ }^{\prime}, 6-$ diamidino-2-phenylindole (DAPI) in dimethyl sulfoxide.
Dilute the stock $1: 1,000$ in staining wash buffer to give a $5 \mu \mathrm{g} / \mathrm{mL}$ DAPI working solution.

10. Peroxidase block: Add $0.3 \%(v / v)$ hydrogen peroxide to staining wash buffer. Store at $4{ }^{\circ} \mathrm{C}$ until use. Keep in the dark to prevent decomposition.

11. ABC-HRP Kit:

1. HRP-conjugated secondary antibody: 1 drop of biotinylated rabbit anti-goat $\lg \mathrm{G}$ in $5 \mathrm{~mL}$ of staining buffer. Store at $4{ }^{\circ} \mathrm{C}$ for up to two weeks.

NOTE: The kit/secondary used will depend on the host species of the primary antibody.

2. Avidin-biotin complex $(A B C)$ reagent: 2 drops of reagent $A$ and 2 drops of reagent $B$ in $5 \mathrm{~mL}$ of staining wash buffer. $A B C$ reagent should be prepared at least $30 \mathrm{~min}$ prior to use. Store at $4{ }^{\circ} \mathrm{C}$ for up to two weeks.

12. Peroxidase substrate: 3 drops of reagent 1,2 drops of reagent 2, 2 drops of reagent 3 and 2 drops of hydrogen peroxide in $5 \mathrm{~mL}$ of $\mathrm{dH}_{2} \mathrm{O}$. Peroxidase substrate should be freshly prepared immediately before use and cannot be stored.

\section{Preparation of skin for wounding}

NOTE: These steps should be performed in a class II laminar flow biosafety cabinet.

1. Collect the skin in holding media and transport to the BSL-2 cabinet.

2. Place the skin dermis side down within a $90 \mathrm{~mm}$ sterile Petri dish and remove adipose tissue with sterile scissors. 
3. Place the skin in a $50 \mathrm{~mL}$ tube containing $25 \mathrm{~mL}$ of HBSS with antibiotics for $10 \mathrm{~min}$ at RT. Shake intermittently to remove any residual blood and adipose tissue.

4. Repeat step 2.3 using a new $50 \mathrm{~mL}$ tube.

5. Place the skin in a fresh $50 \mathrm{~mL}$ tube containing $25 \mathrm{~mL}$ of HBSS, this time without antibiotics for $10 \mathrm{~min}$ at RT. Shake as in step 2.3.

6. Perform a final skin rinse by placing skin in a new tube with $25 \mathrm{~mL}$ of DPBS. The skin is now ready to wound.

\section{Creating ex vivo human skin wounds}

NOTE: These steps should be performed in a class II laminar flow biosafety cabinet.

1. Prepare the skin culture dishes prior to wounding. In a $60 \mathrm{~mm}$ Petri dish, stack two sterile absorbent pads and add $4 \mathrm{~mL}$ of human skin media via the side of the dish. Place a sterile nylon filter membrane onto the absorbent pad stack.

NOTE: Skin media can be altered depending on the required treatment conditions. Up to three wound explants may be cultured on each stack.

2. Dry the dermal side of the skin on sterile gauze in a 90 $\mathrm{mm}$ Petri dish to remove residual DPBS.

NOTE: This prevents the skin from sliding around when wounding.

3. Place the skin dermis side down on a clean $90 \mathrm{~mm}$ Petri dish lid and dab the epidermis dry with fresh sterile gauze.

NOTE: It is easier to wound the skin in a Petri dish lid than the base. Subsequent work should be carried out quickly to prevent the skin drying out.
4. Holding the skin taut, press a $2 \mathrm{~mm}$ biopsy punch against the skin and twist gently. Do not punch entirely through the skin.

NOTE: Partial thickness wounds are designed to punch through the epidermis and partially into the dermis. There may be donor-to-donor and site-to-site variability in the force required to create the partial thickness wound.

5. Use curved toothed tissue forceps to pick up each side of the $2 \mathrm{~mm}$ wound and hook curved iris scissors under the $2 \mathrm{~mm}$ wound to cut it out uniformly.

6. Biopsy around the central $2 \mathrm{~mm}$ wound using a $6 \mathrm{~mm}$ biopsy punch to create a $6 \mathrm{~mm}$ explant with a partial thickness $2 \mathrm{~mm}$ wound in the center.

NOTE: A $6 \mathrm{~mm}$ biopsy punch may be used to score the skin to mark out where each $2 \mathrm{~mm}$ wound should be. Take care not to pierce through the tissue entirely. Create wound explants in a honeycomb pattern to reduce wastage.

7. Place wound explants epidermis side up on the nylon filter membrane stack (prepared in step 3.1).

NOTE: When handling wound explants, be careful not to damage the central wound. Use small forceps and pick up each explant at opposite sides.

8. Incubate wounds at $32-37^{\circ} \mathrm{C}$ and $5 \% \mathrm{CO}_{2}$ in a humidified atmosphere (90-95\%) for 1-7 days. Replace the media every 2-3 days.

\section{Whole-mount staining of ex vivo wounds}

NOTE: This section describes immunofluorescence and immunoperoxidase staining methods. Mix all reagents well before use.

1. Fluorescent staining method 
1. Collect wound explants in $1.5 \mathrm{~mL}$ microcentrifuge tubes containing $500 \mu \mathrm{L}$ of skin fixative and incubate at $4{ }^{\circ} \mathrm{C}$ overnight.

NOTE: The fixative used in this protocol works well for the described antibodies. Optimization will be required for other antibodies. Tissue fixation longer than $24 \mathrm{~h}$ may lead to over-fixation.

2. The following day remove the fixative and replace with $1 \mathrm{~mL}$ of staining wash buffer. Biopsies can be stored in staining wash buffer at $4{ }^{\circ} \mathrm{C}$ up to 2 weeks prior to staining.

NOTE: For all wash buffer steps, use a serological pipette or pipette tip, taking care not to damage the wound.

3. Aspirate the staining wash buffer and perform one more rinse with $1 \mathrm{~mL}$ of staining wash buffer.

4. Calculate the amount of blocking buffer required for steps 4.1.5-4.1.6 (number of samples $\times 300 \mu \mathrm{L}=$ amount of blocking buffer in $\mu \mathrm{L}$ ). Make extra buffer if required.

5. Add $150 \mu \mathrm{L}$ of blocking buffer to each sample and incubate for $1 \mathrm{~h}$ at RT. For all staining steps, ensure each sample is sufficiently covered and that there are no bubbles covering the biopsy wound surface. NOTE: This step onwards can be performed in 1.5 $\mathrm{mL}$ microcentrifuge tubes or in a 48 well plate. If using a 48 well plate, incubate the wounds face down in each well.

6. Dilute the primary antibody in the remaining blocking buffer.

NOTE: Anti-mouse keratin 14 (K14) diluted 1:1,000 in blocking buffer works well. Optimize this step for use with other antibodies or multiple probes.
7. Aspirate the blocking buffer and add $150 \mu \mathrm{L}$ primary antibody per well/microcentrifuge tube. Incubate wound explants in primary antibody at $4{ }^{\circ} \mathrm{C}$ overnight.

8. The next day, aspirate the primary antibody and rinse in staining wash buffer containing $0.2 \%$ sodium azide for $1 \mathrm{~h}$ at RT (500 $\mu \mathrm{L}$ per sample).

9. Perform three more rinsing steps using staining wash buffer (30 min per wash, $500 \mu \mathrm{L}$ per sample).

10. Dilute the fluorescently conjugated secondary antibody in staining wash buffer (e.g., goat antimouse 488 at 1:400 dilution).

11. Calculate the required amount of secondary antibody (number of samples $\times 150 \mu \mathrm{L}=$ amount in $\mu \mathrm{L})$.

12. Add $150 \mu \mathrm{L}$ of secondary antibody to each well/ microcentrifuge tube. Incubate for $1 \mathrm{~h}$ at RT. Perform incubation steps $4.1 .10-4.1 .16$ in the dark as the secondary antibody is light sensitive.

NOTE: This step can be performed at $4{ }^{\circ} \mathrm{C}$ overnight if required. Optimize the concentration of secondary antibody required for adequate signal and limited background staining.

13. Remove the secondary antibody and perform $3 x$ 30 min rinses with staining wash buffer $(500 \mu \mathrm{L}$ per sample).

14. Discard the leftover wash buffer and calculate the amount of DAPI working solution required (as per step 4.1.11).

15. Counterstain each explant with $150 \mu \mathrm{L}$ of DAPI working solution for 10 min at RT. 
NOTE: DAPI will stain cell nuclei blue. Hoechst dye can be used as an alternative to DAPI.

16. Perform two final 30 min washes with staining wash buffer (500 $\mu \mathrm{L}$ per sample). Biopsies can be stored in staining wash buffer at $4{ }^{\circ} \mathrm{C}$ in the dark up to two weeks prior to imaging.

2. Brightfield staining method.

1. Perform steps $4.1 .1-4.1 .3$.

2. Quench endogenous peroxidase activity with peroxidase block at $4{ }^{\circ} \mathrm{C}$ overnight.

NOTE: This step is important when using an HRP-conjugated antibody to reduce non-specific background staining from the tissue. Highly vascularized tissue will contain more endogenous peroxidase activity.

3. Discard the peroxidase block and rinse twice for 30 min in staining wash buffer.

4. Perform steps $4.1 .4-4.1 .8$.

NOTE: Washes after step 4.1 .7 are particularly important to remove sodium azide from the samples. If sodium azide is not adequately removed, it will inactivate the HRP and interfere with staining detection.

5. Add $150 \mu \mathrm{L}$ HRP-conjugated secondary antibody to each well/microcentrifuge tube and incubate overnight at $4{ }^{\circ} \mathrm{C}$ or $1 \mathrm{~h}$ at RT.

6. Remove the secondary antibody and perform $3 \times 30$ min washes in staining wash buffer.

7. Add $150 \mu \mathrm{L} \quad \mathrm{ABC}$ reagent to each well/ microcentrifuge tube and incubate overnight at $4{ }^{\circ} \mathrm{C}$ or 1 hour at RT.
8. Aspirate the $A B C$ reagent and perform $3 \times 30 \mathrm{~min}$ washes in staining wash buffer.

9. Add $150 \mu \mathrm{L}$ peroxidase substrate to one explant and determine the time required to detect a noticeable color change.

NOTE: Choose a sample where strong staining is expected. In this case, a red ring to show the migrating epidermis (K14). 3,3'-diaminobenzidine-4, or any other appropriate chromogenic substrate, may be used as a replacement for this peroxidase substrate.

10. Once a color change is observed, remove the peroxidase substrate, and replace with $1 \mathrm{~mL}$ of $\mathrm{dH}_{2} \mathrm{O}$.

11. Repeat the peroxidase substrate detection for the other explants, incubating for the time determined in step 4.2.11.

12. Rinse all explants with $1 \mathrm{~mL}$ of $\mathrm{dH}_{2} \mathrm{O}$ to remove residual peroxidase substrate. Although explants may be stored up to one week at $4{ }^{\circ} \mathrm{C}$ prior to imaging, it is better to image them as soon as possible to prevent leaching of the peroxidase substrate into the $\mathrm{dH}_{2} \mathrm{O}$ over time.

\section{Imaging and quantification}

1. Fluorescent imaging

NOTE: Fluorescent imaging is performed using a confocal laser-scanning microscope. However, an inverted fluorescent microscope may be sufficient for acquiring $2 \mathrm{D}$ images to quantify wound closure rates. When selecting secondary antibodies, ensure that the chosen fluorochromes are compatible with the excitation 
and emission spectra of the microscopy equipment available.

1. Use a confocal laser-scanning microscope equipped with a $2.5 x, 10 x$ and $20 x$ objective, $x-y-z$ motorized stage, digital camera, and acquisition software. Switch on the transmitted light detector (TPMT) to allow easy visualization of each biopsy and to enable measurement of total wound closure. Alternatively, measure each wound via brightfield microscopy following fluorescence imaging.

2. Place a $60 \mathrm{~mm}$ Petri dish base on the imaging platform and add a thin layer (around $1 \mathrm{~mL}$ ) of DPBS. NOTE: If too much DPBS is used, the biopsy will move around during imaging. Alternatively use a 48 well plate if a plate holder is available.

3. Use small tissue forceps to transfer wound explants from wells/microcentrifuge tubes to the Petri dish containing DPBS. Place the biopsy wound side down in the Petri dish.

4. Use the eyepiece and fluorescent lamp to locate and focus on the wound. If bubbles are trapped under the sample in the field of view, pick up the wound with tissue forceps and reposition.

5. Set up the imaging software, ensuring equal pinhole size between channels for optimum confocality. For this, check the value of one airy unit for each channel and select the largest value. Select scan speed, image quality and averaging.

NOTE: The fluorochromes of the conjugated secondary antibodies and the chosen counterstain (e.g., DAPI) will dictate the channels required.

6. Switch on the live acquisition software and adjust the laser power and gain of each channel to the levels required to visualize staining. Reduce background noise by increasing the digital offset.

7. Position the wound in the center of the imaging plane.

NOTE: If the wound does not fill the entire image due to using a smaller objective or creating a larger wound, take a panel of images and stitch them together (manually or with a tiling function in the relevant imaging software).

8. Acquire images of the wound biopsies. Use the same imaging settings between explants.

NOTE: Higher power images will allow assessment of tissue structures and cellular marker expression and location.

9. Collect serial Z stacks through the wound, especially where the tissue is not completely flat against the Petri dish. Use analysis software to collapse the Z stack into a single maximum intensity projection image.

2. Brightfield imaging

NOTE: Brightfield imaging of immunoperoxidase stained biopsies can be performed in multiple ways.

1. Inverted microscope imaging: Prepare wound explants for imaging by placing them in a Petri dish/ well as described in steps 5.1.2-5.1.3. Acquire digital images under brightfield illumination on an inverted microscope equipped with a digital camera. Stitch together multiple images if required.

2. Wireless digital microscope imaging: Use a wireless digital microscope connected to a phone or laptop to obtain high quality images in a cost-effective manner. Place explants wound side up onto some tissue and remove any residual $\mathrm{dH}_{2} \mathrm{O}$ (or staining 
wash buffer) from sample storage. Position the wound explant in the center of the microscope field of view. Acquire images using the connected camera.

3. Quantification

NOTE: Percentage wound closure can be quantified in any software that allows freehand shapes to be drawn and measured. ImageJ can be used to perform quantification as follows:

1. Open the image to be quantified in ImageJ software.

2. Use the freehand shape tool to draw around the outside of the re-epithelialized wound where it meets the normal skin. Press M (or Analyze | Measure) to acquire an 'outer' area measurement.

NOTE: The re-epithelialized wound tissue texture differs from normal skin. The images do not need to be scaled prior to this type of analysis.

3. Use the freehand shape tool to draw around the open wound area. This is where the open wound meets the inside edge of the re-epithelializing tissue.

Press M (or Analyze | Measure) to acquire an 'inner' area measurement.

4. Use the following equation to deduce percentage wound re-epithelialization/closure:

$\%$ Closure = (Outer Wound Area - Inner Wound Area) / (Outer Wound Area) x 100

NOTE: Percentage area coverage of antibodies can be deduced in the same way (e.g., $\mathrm{K} 14)$ or as a percentage of the total wound area. Percentage intensity can also provide semi-quantitative information about tissue level expression of markers of interest, while high power imaging presents expression data at the cellular level.

\section{Representative Results}

In this report, we present a novel ex vivo skin wounding and whole-mount staining approach to assess factors that influence the human skin repair response. Figure $1 \mathrm{~A}$ shows a schematic of the procedural pipeline, which can be performed in 3-10 days, depending on wound incubation times. The partial thickness wounds are cultured on membrane stacks at the air : membrane interface and can be collected for whole-mount staining, embedded in paraffin or OCT medium for general histology, or frozen in liquid nitrogen for biochemical analysis (Figure 1B). We generally create $2 \mathrm{~mm}$ partial thickness wounds within the center of $6 \mathrm{~mm}$ explants. However, the size of the wound and surrounding explant may be altered depending on requirements. The whole-mount procedure has been successfully adapted for both immunoperoxidase and immunofluorescence staining methods (Figure 1C).

Immunofluorescence allows for the probing of tissue with multiple antibodies. For this, we advise using primary antibodies raised in different species, and species-matched fluorescently conjugated secondary antibodies to limit crossspecies reactivity. Antibody concentrations and incubation times will need to be optimized. If background staining is observed, reduce antibody concentrations, increase wash steps, and add blocking buffer to the secondary antibody. Fresh tissue viability can be directly assessed with commercial viability dyes (see Table of Materials). We also show that tissue may be fixed post viability staining and successfully imaged when it is practically suitable (Figure 1D). 
A)

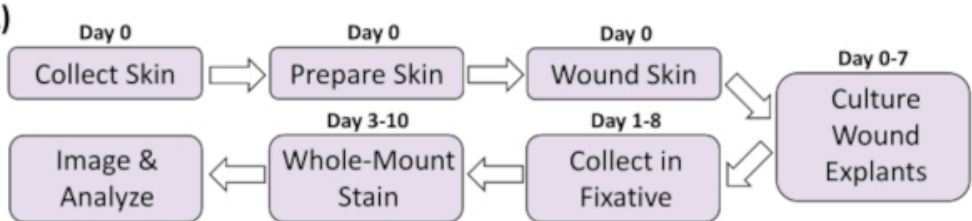

B)

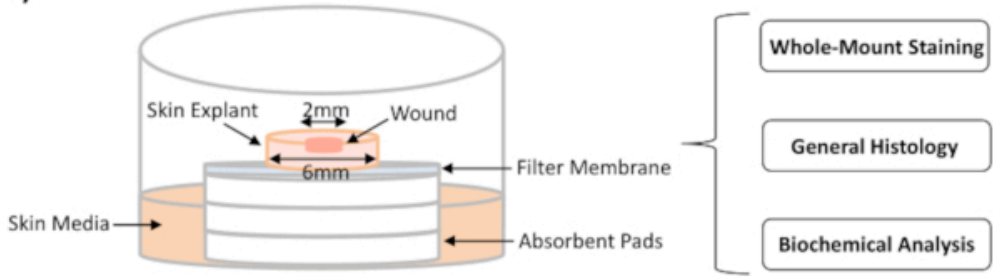

C)
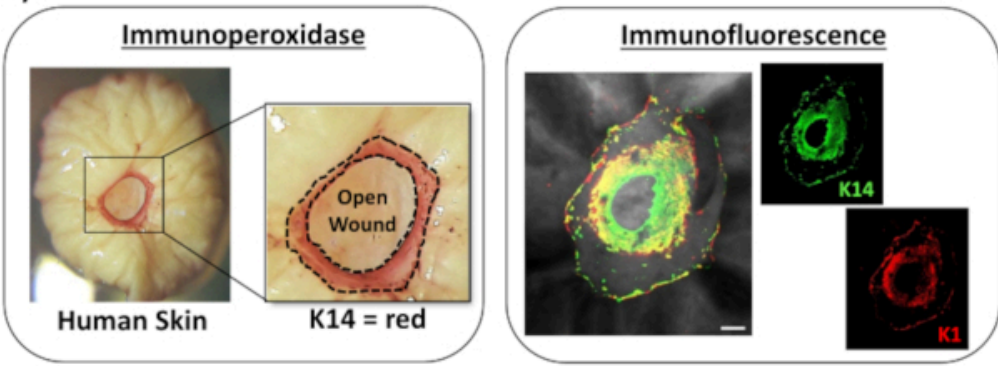

D)
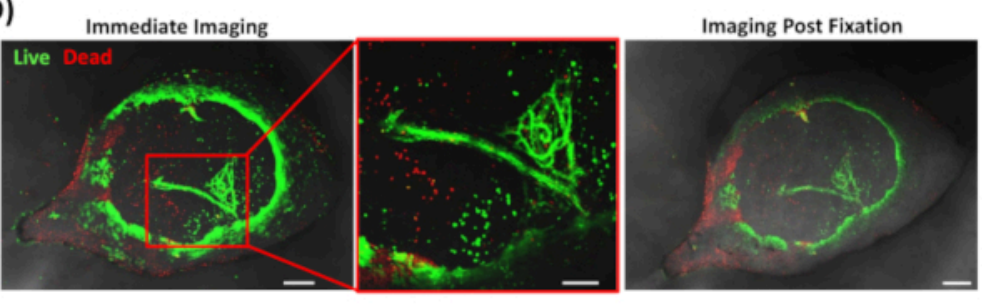

Figure 1: The human ex vivo wounding and whole-mount staining approach. (A) Pipeline depicting the procedural workflow from collecting skin and performing ex vivo wounding, to staining tissue and analyzing data. (B) Diagram demonstrating the human ex vivo skin wound culture system with analyses routinely performed on the tissue. (C) Wholemount staining can be employed using both immunoperoxidase and immunofluorescence techniques. $\mathrm{K} 14=\mathrm{keratin} 14$. (D) Live tissue may be stained with commercial viability dyes and imaged successfully post fixation. Bar $=100 \mu \mathrm{m}$. This staining was performed in non-diabetic skin. Please click here to view a larger version of this figure.

The most widely applicable use for whole-mount staining of wounds is to determine wound closure rate in a more reproducible manner than can be provided via histological sectioning. Percentage closure was quantified as percentage re-epithelialization of the wound surface, as demonstrated in Figure 2A. Percentage area coverage of specific markers can be measured from the total wound area or as a percentage of the re-epithelialized wound. We characterized healing in healthy (non-diabetic) versus diabetic skin across a time course of seven days, collecting wounds at each day postwounding (representative images, Figure 2B). Healthy skin wounds closed over time as expected, with full closure 
observed in most samples by day 4-5. On the contrary, diabetic skin wounds failed to close fully within the sevenday analysis period (Figure 2C). A significant delay in wound closure was observed between healthy and diabetic skin wounds when comparing healing rates at each time-point post-injury $(P<0.001$ to day $6, P<0.05$ at day 6 and $P<0.05$ to $P<0.001$ at day 7 ).

Following assessment of overall wound closure rates; we measured the percentage of the entire wound area (outer area in Figure 2A) where K14 positive cells could be visualized (green staining in Figure 2B). Interestingly, we observed that in healthy ex vivo skin wounds, K14 staining peaked at day 2 and then rapidly declined (significance at each time-point versus the day 2 peak, Figure 2D). This is likely reflecting re-formation of the early epidermal barrier, excluding K14 antibody penetration through differentiated epidermal layers (see Figure 2E schematic). During the reepithelialization process, basal layer (K14+ve) keratinocytes migrate inwards over the open wound such that the epidermis closer to the outer wound edge forms earlier than the epidermis closer to the inner wound edge (migrating front). While the front edge of the newly formed epidermis continues to migrate to close the remaining open wound, the outer edge epidermis begins to differentiate to reform the other epidermal layers. In early healing, we would thus expect to see most of the re-epithelialized area consists of basal (K14+ve) cells, while in later repair K14 staining is lost as the epidermis differentiates from the outside inwards (see whole-mount images in Figure 2E). Therefore, the decline in K14 staining shown in Figure 2D (downward arrows) correlates with increased epidermal differentiation. Interestingly, visible K14 staining peaked earlier in healthy (day 2) versus diabetic (day 4) wounds, further demonstrating that re-epithelialization and subsequent epidermal differentiation are delayed in diabetic skin wounds. 
A)

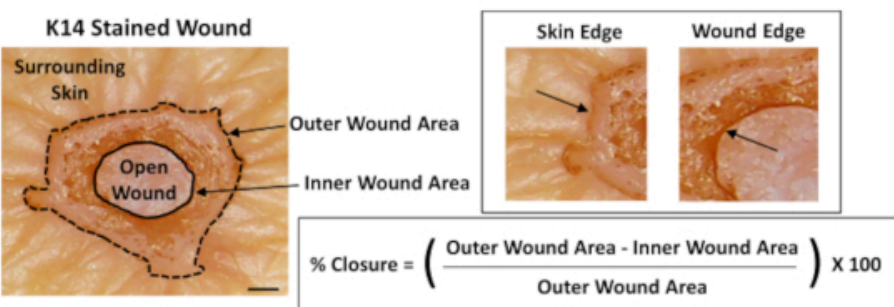

B)

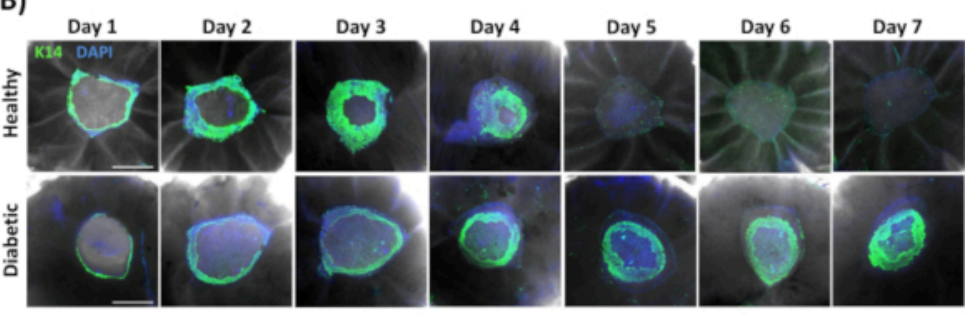

C)

D)

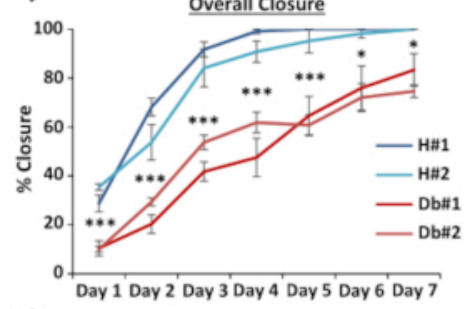

Wound K14

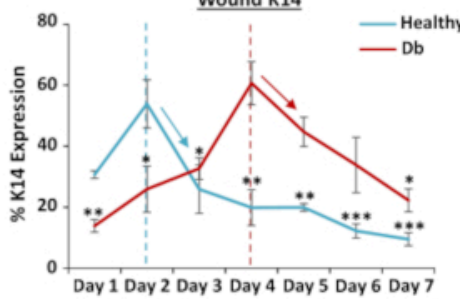

E)

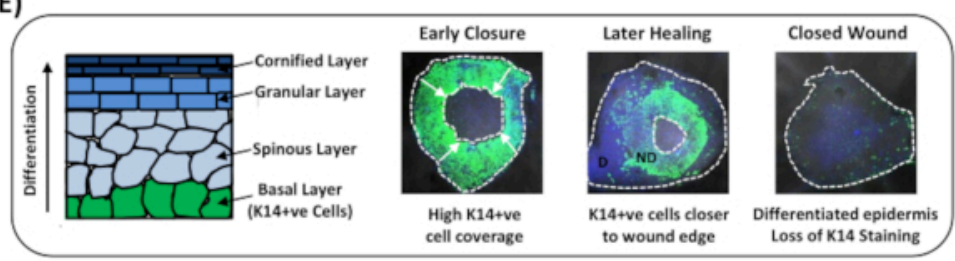

Figure 2: Whole-mount staining reveals perturbed healing rates in diabetic versus healthy skin. (A) The method used to quantify wound closure from outer and inner wound measurements. Brightfield images show keratin 14 (K14) staining in red. Bar $=300 \mu \mathrm{m}$. (B) Representative images of healing over time (day post-wounding) in healthy and diabetic skin. Bar $=$ $500 \mu \mathrm{m} . \mathrm{K} 14$ = green. DAPI = blue nuclei. (C) Quantification of wound closure rates (percentage re-epithelialization) showing that ex vivo wounds from healthy skin close significantly faster than ex vivo wounds from diabetic skin. $\mathrm{H}=\mathrm{healthy}$. $\mathrm{Db}=$ diabetic. (D) Percentage K14 staining peaks earlier in healthy versus diabetic skin and then declines in line with increased epidermal differentiation (down arrows). (E) K14 (basal epidermal cell) staining is lost as the epidermis differentiates. D = differentiated. ND = not differentiated. White dotted lines depict inner and outer wound edges. White arrows $=$ direction of migration. $\mathrm{n}=6$ wounds per donor, per time point. Mean $+/-\mathrm{SEM} .{ }^{*}=P<0.05,{ }^{* *}=P<0.01$ and ${ }^{* * *}=P<0.001$. Healthy and diabetic compared at each healing time point in $\mathbf{C}$ ( $P$ value for least significant comparison). Temporal change in $\mathrm{K} 14$ staining compared to peak for each donor in $\mathbf{D}$. Please click here to view a larger version of this figure. 
We next used whole-mount staining to explore tissue expression and localization of other wound-relevant markers in non-diabetic skin (Figure 3). All antibodies used and their working concentrations are provided in the Table of Materials. Blood vessels in the open wound stained positively with alpha smooth muscle actin (a-SMA) antibody, used in combination with $\mathrm{K} 14$ to delineate the epidermal edges in lower power images (Figure 3A). The dermal matrix was stained with antibodies against collagen type I (COL 1) and fibronectin (Fn). Here collagen was observed as abundant thick fibers while fibronectin fibers were sparse, wavy, and thin (Figure 3A). Our whole-mount staining approach is also able to provide cell level resolution of staining, as demonstrated for K14-positive keratinocytes (Figure 3B).

Finally, we show that human ex vivo wounds possess resident immune cells, with Langerhans cells detected around newly formed epidermis at day 3 post-wounding (Figure $3 \mathrm{C}$ ). Indeed, these results suggest that whole-mount staining may be used to investigate key features of the healing response including inflammation, proliferation, and the extracellular matrix (Figure 4A). Taken together, our data reveal that the combined ex vivo skin wounding and whole-mount staining procedure is a valid method to assess various aspects of healthy and diabetic (pathological) human skin repair. 

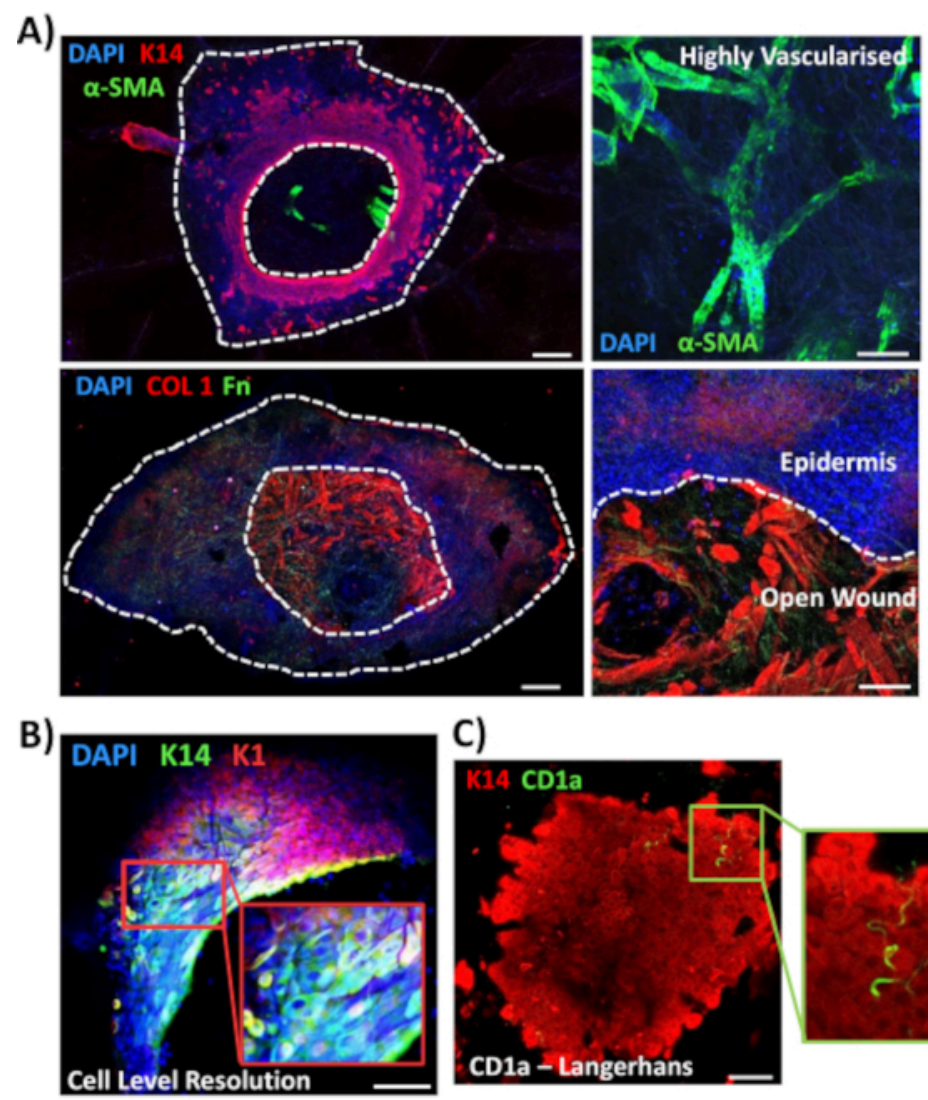

C)
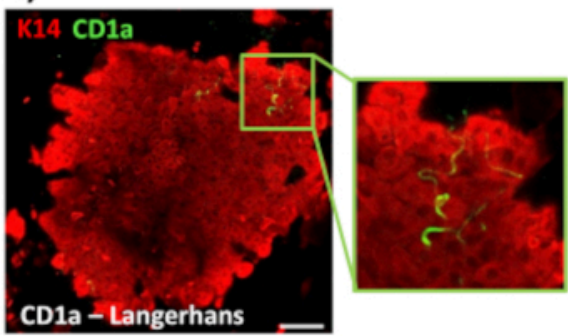

Figure 3: Optimization of the whole-mount staining approach for use with other antibodies. (A) Blood vessels were stained with alpha smooth muscle actin ( $\alpha-S M A$, green) and keratin $14(\mathrm{~K} 14$, red), while matrix fibers were stained with collagen I (COL 1, red) and fibronectin (Fn, green). (B) The whole-mount procedure provides up to cell level resolution of localization (K14, green; K1, red). (C) CD1a+ve Langerhans cells (green) observed in newly formed epidermis. DAPI = blue nuclei. Bar $=100 \mu \mathrm{m}$. White dotted lines show inner and outer wound edges and separate wound from epidermis. This staining was performed in non-diabetic skin. Please click here to view a larger version of this figure. 
A)

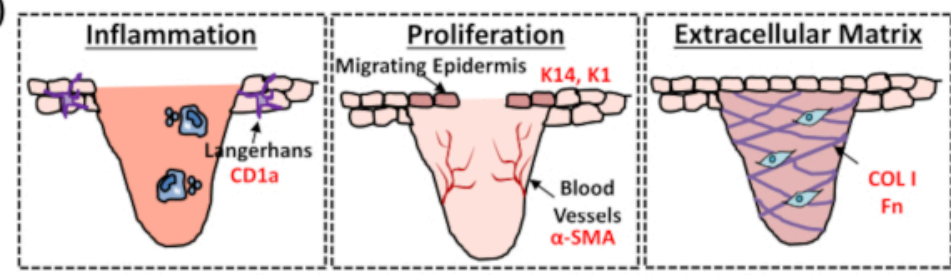

B)

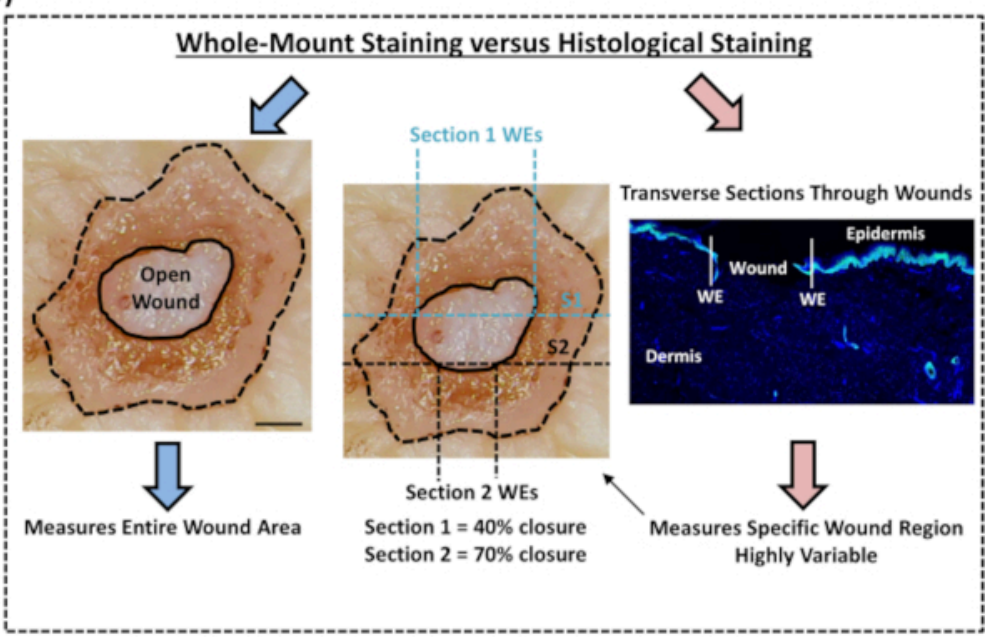

Figure 4: Validity of the whole-mount staining procedure for assessing wound healing. (A) Illustration depicting how the whole-mount staining technique can evaluate wound-relevant processes. Antibodies used = red text. K14 = keratin 14 . COL $1=$ collagen $1 . \mathrm{Fn}=$ fibronectin. $(\mathrm{B})$ The whole-mount staining procedure (blue arrows) introduces less variability to wound closure measurements than standard histological analysis (red arrows). S1 $=$ section 1. WE $=$ wound edge. Bar $=300$ $\mu \mathrm{m}$. This staining was performed in non-diabetic skin. Please click here to view a larger version of this figure.

\section{Discussion}

In this experimental protocol, we describe an optimized method for evaluating wound closure in human ex vivo skin using whole-mount tissue staining. This is an important resource to allow critical evaluation of potential wound treatments, and to provide better understanding of the human wound repair response. We have published healing assessment in ex vivo skin wounds previously ${ }^{12,13}$, yet in these reports the whole-mount staining approach was not used to measure wound closure. Whole-mount staining is far easier and requires less technical experience than standard histology, which involves paraffin or OCT embedding and sectioning of samples. The whole-mount procedure also reduces experimental variability, allowing quantification of the entire wound and not just a single transverse section at a defined position within the tissue (see Figure 4B for comparative illustration). We fully support the importance of quantifying healing of the entire non-symmetrical wound structure, as clearly outlined by Rhea and Dunnwald for murine acute wounds ${ }^{14}$. These authors showed the importance of serially sectioning in vivo excisional wounds for reproducible and precise measurements of wound morphology. Serial sectioning could equally be applied to 
human ex vivo wounds; however, for accurate quantification of wound closure and re-epithelialization, high throughput whole-mount staining should be the preferred method. We note that this whole-mount staining protocol should also be compatible with subsequent processing (wax or OCT) for traditional histological analysis.

Whole-mount staining is not without disadvantages. While it affords higher reproducibility in wound healing experiments, it does require the use of more tissue for analysis than standard histological techniques. This may be an issue where tissue access is limited, particularly where multiple antibodies need to be assessed. An alternative approach would be to employ an incisional wounding method where wound width is relatively uniform and variability is reduced (as shown in mouse and human wounds ${ }^{15,16}$ ). However, excisional wounds remain more applicable to most pathological wound types $^{17}$.

In this study, $2 \mathrm{~mm}$ partial thickness wounds were created within the center of $6 \mathrm{~mm}$ skin explants. This method may be optimized for alternative excisional wound and explant sizes at different skin depths ${ }^{18}$. In addition, the force required to generate wounds will vary between donors, where aged skin will require less force to biopsy. We would also avoid using skin displaying prominent stretch marks or other structural alterations. We have validated a range of antibodies to consider different aspects of the ex vivo healing response. This protocol may also be used with other skin-relevant antibodies, where antibody concentrations and incubation times will need to be optimized. Nevertheless, we believe our protocol is most suited to absolute quantification of total wound closure, followed by spatial assessment of specific proteins of interest. While whole-mount provides reduced resolution of immunolocalization versus standard histological analysis of tissue sections, it provides additional $3 \mathrm{D}$ information that is missing from standard 2D histology.

One caveat of assessing healing in ex vivo skin versus in vivo models is that it lacks a systemic response. An important aspect of wound repair is inflammation and subsequent tissue granulation, which is caused by an influx of inflammatory cells and endothelial cells from the vasculature $^{19}$. Despite this limitation, ex vivo skin still provides a better recapitulation of clinical healing than cell-based wound assays. In vitro experiments in general involve single cell type monolayers or co-cultures grown on tissue culture plastic, whereas ex vivo skin provides a native environment to explore cell behavior. More recently, a number of skin equivalent systems have emerged, where skin is grown in a laboratory setting from artificial matrix and isolated skin cells 20,21 . Although these models mimic human skin better than most in vitro approaches, they still do not fully simulate the native tissue environment and are generally too fragile to injure reproducibly. Additionally, we (and others) have demonstrated that ex vivo human skin tissue retains resident immune cells, which will no doubt contribute to repair 22,23 . Future work should now focus on extending the viability and immunocompetency of the ex vivo model for late-stage healing assessment ${ }^{24}$. One option is further advancement of promising organ-on-achip technologies capable of prolonging tissue viability and maintaining native skin architecture for up to two weeks in culture $^{25}$. Ex vivo models have also begun to consider the importance of the skin inflammatory response by successfully incorporating immune cells, such as neutrophils, into the host tissue $^{26}$ or injecting host tissue with antibodies to elicit an immune reaction ${ }^{27}$. We expect that these findings will pave 
the way for development of more refined and translatable methods in the future.

A major benefit of using ex vivo skin to measure wound closure is the ability to compare healing rates in healthy (e.g., non-diabetic) versus pathological (e.g., diabetic or aged) tissue. Here we showed that re-epithelialization and barrier formation are indeed impaired in diabetic versus healthy ex vivo wounds. Indeed, this provides a route for pre-clinical assessment of pathological repair, where ageing and diabetes are major risk factors for developing chronic wounds ${ }^{1}$. While in vitro pathological models exist, such as cells isolated from aged and diabetic tissue, or cells cultured in high glucose to mimic hyperglycemia 28,29 , these cells can quickly lose their phenotype once removed from the in vivo microenvironment. An important component of the extrinsic pathological healing environment is the dermal matrix, which is altered in both ageing and diabetes ${ }^{30}$. Indeed, this perturbed matrix affects the behavior of resident and naïve fibroblasts ${ }^{31,32}$. Thus, the importance of studying cells in their host tissue environment cannot be underestimated.

In summary, our protocol provides an important platform to quantify human wound re-epithelialization, explore regulatory factors and to test the validity and efficacy of potential therapeutics $^{12,13}$. While pre-clinical testing does still require in vivo approaches, a combined strategy using ex vivo human tissue and in vivo murine wounding should refine the preclinical pathway, reducing animal use while increasing crossspecies translatability.

\section{Disclosures}

The authors declare no conflicts of interest.
We would like to thank Mr Paolo Matteuci and Mr George Smith for providing patient tissue. We are also grateful to Miss Amber Rose Stafford for assisting with tissue collection and the Daisy Appeal for providing laboratory facilities.

\section{References}

1. Lindholm, C., Searle, R. Wound management for the 21st century: combining effectiveness and efficiency. International Wound Journal. 13, 5-15 (2016).

2. Guest, J. F. et al. Health economic burden that different wound types impose on the UK's National Health Service. International Wound Journal. 14 (2), 322-330 (2017).

3. Guest, J. F., Fuller, G. W., Vowden, P. Diabetic foot ulcer management in clinical practice in the UK: costs and outcomes. International Wound Journal. 15 (1), 43-52 (2018).

4. López-Valverde, M. E., et al. Perioperative and longterm all-cause mortality in patients with diabetes who underwent a lower extremity amputation. Diabetes Research and Clinical Practice. 141, 175-180 (2018).

5. Wilkinson, H. N., Hardman, M. J. The role of estrogen in cutaneous ageing and repair. Maturitas. 103, 60-64 (2017).

6. Frykberg, R. G., Banks, J. Challenges in the treatment of chronic wounds. Advances in Wound Care. 4 (9), 560-582 (2015).

7. Wilkinson, H. N., Hardman, M. J. Wound healing: cellular mechanisms and pathological outcomes. Open Biology. 10 (9), 200223 (2020).

\section{Acknowledgments}


8. Ansell, D. M., Holden, K. A., Hardman, M. J. Animal models of wound repair: Are they cutting it? Experimental Dermatology. 21 (8), 581-585 (2012).

9. Elliot, S., Wikramanayake, T. C., Jozic, I., Tomic-Canic, M. A modeling conundrum: murine models for cutaneous wound healing. Journal of Investigative Dermatology. 138 (4), 736-740 (2018).

10. Mazio, C. et al. Pre-vascularized dermis model for fast and functional anastomosis with host vasculature. Biomaterials. 192, 159-170 (2019).

11. Wilkinson, H. N., Iveson, S., Catherall, P., Hardman, M.J. A novel silver bioactive glass elicits antimicrobial efficacy against Pseudomonas aeruginosa and Staphylococcus aureus in an ex vivo skin wound biofilm model. Frontiers in Microbiology. 9, 1450 (2018).

12. Wilkinson, H. N. et al. Elevated local senescence in diabetic wound healing is linked to pathological repair via CXCR2. Journal of Investigative Dermatology. 139 (5), 1171-1181 (2019).

13. Wilkinson, H. N. et al. Tissue iron promotes wound repair via M2 macrophage polarization and the chemokine (CC motif) ligands 17 and 22. The American Journal of Pathology. 189 (11), 2196-2208 (2019).

14. Rhea, L., Dunnwald, M. Murine excisional wound healing model and histological morphometric wound analysis. Journal of Visualized Experiments. 162, e61616 (2020).

15. Ansell, D. M., Campbell, L., Thomason, H. A., Brass, A., Hardman, M.J. A statistical analysis of murine incisional and excisional acute wound models. Wound Repair and Regeneration. 22 (2), 281-287 (2014).

16. Rizzo, A. E., Beckett, L. A., Baier, B. S., Isseroff, R. R. The linear excisional wound: an improved model for human ex vivo wound epithelialization studies. Skin Research and Technology. 18 (1), 125-132 (2012).

17. Olsson, M., et al. The humanistic and economic burden of chronic wounds: a systematic review. Wound Repair and Regeneration. 27 (1), 114-125 (2019).

18. Mendoza-Garcia, J., Sebastian, A., Alonso-Rasgado, T., Bayat, A. Optimization of an ex vivo wound healing model in the adult human skin: Functional evaluation using photodynamic therapy. Wound Repair and Regeneration. 23 (5), 685-702 (2015).

19. Brownhill, V. R., et al. Pre-clinical assessment of singleuse negative pressure wound therapy during in vivo porcine wound healing. Advances in Wound Care. "In Press" (2020).

20. Diekmann, J., et al. A three-dimensional skin equivalent reflecting some aspects of in vivo aged skin. Experimental Dermatology. 25 (1), 56-61 (2016).

21. Vidal Yucha, S. E., Tamamoto, K. A., Nguyen, H., Cairns, D. M., Kaplan, D. L. Human skin equivalents demonstrate need for neuro-immuno-cutaneous system. Advanced Biosystems. 3 (1), 1800283 (2019).

22. Dijkgraaf, F. E. et al. Tissue patrol by resident memory CD8+ T cells in human skin. Nature Immunology. 20 (6), 756-764 (2019)

23. He, X., de Oliveira, V. L., Keijsers, R., Joosten, I., Koenen, H. J. Lymphocyte isolation from human skin for phenotypic analysis and ex vivo cell culture. Journal of Visualized Experiments. (110), e52564 (2016).

24. Pupovac, A. et al. Toward immunocompetent 3D skin models. Advanced Healthcare Materials. 7 (12), 1701405 (2018). 
25. Ataç, B. et al. Skin and hair on-a-chip: in vitro skin models versus ex vivo tissue maintenance with dynamic perfusion. Lab on a Chip. 13 (18), 3555-3561 (2013).

26. Kim, J. J. et al. A microscale, full-thickness, human skin on a chip assay simulating neutrophil responses to skin infection and antibiotic treatments. Lab on a Chip. 19 (18), 3094-3103 (2019).

27. Jardet, C. et al. Development and characterization of a human Th17-driven ex vivo skin inflammation model. Experimental Dermatology. 29 (10), 993-1003 (2020).

28. Chen, J. L. et al. Metformin attenuates diabetesinduced tau hyperphosphorylation in vitro and in vivo by enhancing autophagic clearance. Experimental Neurology. 311, 44-56 (2019).

29. Demirovic, D., Rattan, S. I. Curcumin induces stress response and hormetically modulates wound healing ability of human skin fibroblasts undergoing ageing in vitro. Biogerontology. 12 (5), 437-444 (2011).

30. Wilkinson, H. N., Hardman, M. J. Wound senescence: A functional link between diabetes and ageing? Experimental Dermatology. 30 (1), 68-73 (2020).

31. Fisher, G. J. et al. Collagen fragmentation promotes oxidative stress and elevates matrix metalloproteinase-1 in fibroblasts in aged human skin. The American Journal of Pathology. 174 (1), 101-114 (2009).

32. Quan, T., Little, E., Quan, H., Voorhees, J. J., Fisher, G. J. Elevated matrix metalloproteinases and collagen fragmentation in photodamaged human skin: impact of altered extracellular matrix microenvironment on dermal fibroblast function. Journal of Investigative Dermatology. 133 (5), 1362 (2013). 